\title{
Effects of cigarette smoking on the evolution of hearing loss caused by industrial noise
}

\author{
Abbate Carmelo ${ }^{1 *}$, Giorgianni Concetto ${ }^{1}$, Zirilli Agata ${ }^{2}$, Tringali Maria Antonietta ${ }^{1}$, D'Arrigo \\ Graziella ${ }^{2}$, Brecciaroli Renato ${ }^{1}$, Abbate Adriana1, Salmaso Luigi ${ }^{3}$ \\ ${ }^{1}$ Department of Social Medicine of Messina University, Section of Occupational Medicine, Messina, Italy; *Corresponding Author: \\ abbatec@unime.it \\ ${ }^{2}$ Statistics Department, Messina University, Messina, Italy \\ ${ }^{3}$ Statistics Department, Vicenza University, Vicenza, Italy
}

Received 11 June 2010; revised 6 July 2010; accepted 26 July 2010.

\begin{abstract}
The few studies evaluating the changes caused by cigarette smoking on hearing loss induced by occupational exposure to noise have reached discordant conclusions. The aim of this study is to investigate the interactions between cigarette smoking and occupational exposure to noise as risk factors in the onset and development of hearing loss. The study was performed on a sample of 557 shipyard workers exposed to noise at an Equivalent Level (Leq) of $93 \mathrm{dBA}$. On the basis of their smoking habits, they were divided into three groups: group (A), non-smokers; group (B), smokers (15-30 cigarettes per day); and group (C), heavy smokers (over 30 cigarettes per day). The study focussed on the audiometric responses of the subjects at the frequencies of $500,1000,2000,3000$ and $4000 \mathrm{~Hz}$. The results were then compared using statistical techniquees (Internal correlation coefficient, exponential model, ANCOVA, NPC test). Comparison of the audiometric responses showed statistically significant differences between the three groups. Non-parametric analysis, performed using the NPC test, highlighted that the interaction between smoking and exposure to noise has an influence on hearing loss at all frequencies, and particularly at high frequencies $(3000-4000 \mathrm{~Hz})$. The data obtained from the examined sample show that smoking and exposure to noise cause an increase in occupational hearing loss and that this is directly related to the number of cigarettes smoked.
\end{abstract}

Keywords: Smoking; Hearing Loss; Noise; Audiometric Test; Non Parametric Test

\section{INTRODUCTION}

Hearing loss as a result of exposure to noise is one of the most frequent pathologies found in workers, and is influenced by a number of parameters, such as intensity of the noise, temporal and spectral patterns, duration of the exposure and susceptibility factors. Controlling these parameters may improve the individual response to noise and evolution of the damage.

One of the factors influencing hearing susceptibility which has attracted growing interest in recent years is cigarette smoking, although the role of smoking in abetting the development of sensory neural hearing loss is controversial, as recent literature shows.

Some authors highlight that smokers have a greater risk of hearing loss than non-smokers [1-3], while others find no connection between the onset of sensory neural hearing loss and cigarette smoking [4,5]. Nomura [6] in a review referring to the period 1966-2003 , mentions 9 studies which report a positive association between smoking and hearing loss, and 6 which reach completely opposite conclusions. The author, however, concludes by supporting the thesis of a positive association between smoking and hearing loss.

Few studies evaluate the effects of the association between smoking, occupational exposure to noise and hearing loss. Palmer et al. [7] hypothesises that exposure to noise in smokers aggravates the degree of sensory neural hearing loss, probably due to the effect that these factors have on the vascular system. In fact, both exposure to noise and smoking cause vasoconstriction in the cochlear vessels with consequent reduced perfusion, increased plasma viscosity and/or possible creation of carboxyhaemoglobin, which aggravate any local hearing loss. Mizoue et al. [8] observes that the hearing loss affects only the cells responsible for the hearing response to high frequencies, since their position at the end of the nutritive arteries makes them more vulnerable to ischaemic damage. 
Dengerink et al. [9], in an experimental study on temporary threshold shifts (TTS) in man, highlights how cigarette smoking modifies the response of hearing to noise exposure, and attributes this effect to carbon monoxide (CO) and nicotine. Ferrite et al. (2005) confirm a synergic effect between cigarette smoking, exposure to noise and age on hearing loss caused by industrial noise. Nomura [10], in a cross-sectional study, carried out on 397 Japanese workers exposed to noise, finds a positive association between smoking, exposure to noise and hearing loss, even if this is often masked by atherosclerotic factors.

Jaruchinda [11] asserts that cigarette smoking influences noise injury in helicopter pilots and aircraft mechanics. Uclide asserts that the combined effects of noise and smoking is not interactive but additive. Recently, Pouryaghoub [12] concludes that smoking can accelerate noise induced hearing loss, but more research is needed to understand the underlying mechanism

Other authors reach opposite conclusions. Pyykko et al. [13] observe that smoking does not affect the incidence of noise-induced hearing loss in 199 forestry workers. Nakashima et al. [14], in a case-control study conducted on 109 subjects, reports that hearing loss is not affected by smoking.

Since the interactions between industrial noise, cigarette smoking and hearing loss are uncertain, this study aims to clarify the relationship between such variables by trying to evaluate the effects of external factors on hearing loss. This information is particularly useful when drawing up prevention programs aimed at protecting workers' hearing. As far as regards smoking, and in the absence of specific studies, the possible effect of the number of cigarettes smoked per day is also taken into consideration.

\section{METHODS}

The study was carried out on male subjects, who had only ever had one job, as shipwrights at a large shipyard for the construction of high-speed boats in southern Italy. The enrolled sample was composed of the entire workforce of 900 men.

Noise measurement, performed in November 2003 according to European directives 89/391/EEC and 86/188/ EEC, showed that all the subjects were exposed to a daily personal exposure level (LEP,d) of $93 \pm 2 \mathrm{dBA}$ of equivalent level. The exposure value reported did not display significant variations compared to the controls previously performed by the company every three years from 1992 onwards, as provided for by Italian legislation regarding occupational exposure to noise. This lays down that the noise risk in working environments and the LEP, $\mathrm{d}$ for each individual worker must be assessed.

The subjects enrolled for the study were selected by applying the following exclusion criteria:

1) previous exposure to neurotoxic drugs

2) frequent use of ototoxic drugs

3) metabolism diseases

4) haematological or neurological diseases

5) acute and chronic ear, nose and throat conditions

6) residence since birth in a Council district other than that in which the subject works

7) alcoholism

8) former smokers, those who had been smoking for under 10 years and subjects whose smoking habits had significantly changed over the years

9) hobbies such as hunting, underwater fishing, frequent visits to discos ( $>$ once per week)

10) work experience with other companies

11) length of service $<10$ years.

All the subjects normally used individual hearing protection devices supplied by the company (earphones and earplugs).

The sample thus selected was composed of 557 subjects, who were divided into three groups on the basis of their smoking habits. The first was composed of non-smokers since birth (Group A), the second (Group B) of smokers (15-30 cigarettes per day for at least 10 years), and the third (Group C) of heavy smokers (over 30 cigarettes per day for at least 10 years). People smoke lass than 14 sigarettes a day have not been taken in consideration because the data is not significant.

The entire sample was given a general medical examination, routine blood tests, otoscopic examination, and a tonal audiometric test after at least 16 hours without exposure to occupational noise.

For the purposes of tonal audiometric assessment, the study took into consideration hearing threshold values at the frequencies of 500, 1000, 2000, 3000 and $4000 \mathrm{~Hz}$.

The study was based on the values recorded by the tonal audiometric traces.

Table 1 shows the sample size, average age and years of service of the subjects, by group:

Table 1. Sample size of the groups and mean values for age and length of service.

\begin{tabular}{cccc}
\hline Group & Sample size & Age $\pm \sigma$ & Length of service $\pm \sigma$ \\
\hline A & 215 & $41.45 \pm 7.81$ & $21.36 \pm 7.57$ \\
B & 194 & $40.77 \pm 8.83$ & $20.15 \pm 8.34$ \\
C & 148 & $40.12 \pm 8.77$ & $20.25 \pm 8.35$ \\
ABC & 557 & $40.98 \pm 8.52$ & $20.77 \pm 8.15$ \\
\hline
\end{tabular}


The aim of the analysis was to assess any significant differences, if they exist, between the audiometric responses of the three groups determined not only by their being smokers or not, but also by the quantity of cigarettes smoked daily.

Statistical analysis - To evaluate whether the audiometric responses of the two ears are interdependent, we used an internal correlation coefficient. An exponential model was also used to describe the trend of audiometric responses in function of the risk index. Moreover, to verify whether the trend of the responses may be expressed with a single function, the ANCOVA (Analysis of Covariance) model was adopted. Lastly, we used an NPC (Non-Parametric Combination) test to compare all the audiometric responses of the three groups for the various risk indexes.

Considering that biological age and the duration of exposure influence audiometric response, and consequently may determine modifications in the individual responses at the various frequencies, we used a risk index [14] which made it possible to evaluate the combined action of the aforementioned variables.

The relation which makes it possible to determine the risk index $\left(I_{r}\right)$ is:

$$
I_{r}=1-\left(\frac{E_{a}-A_{l}}{E_{a}}\right)
$$

where:

$A_{1}$ represents length of service;

$E_{a}$ biological age.

each group, the following type of model was used:

$$
R_{a}=a \cdot b^{x}
$$

where $x=(\text { Age of starting work })^{(\text {Risk index })}$.

\section{RESULTS}

The Table 2 shows the audiometric responses at different frequencies in the three group. Since we measured the audiometric responses of both ears for each subject, we decided to assess whether there was any close interdependence between them. In order to achieve this we established the internal correlation co-efficients between the distributions of the responses of the two ears, for each group and frequency (Table 3).

The internal correlation coefficients all indicate interdependence between the responses of the left and right ear for all frequencies and all groups.

Consequently, analysis may be performed on the responses of either ear, chosen at random, in each subject. The risk index showed the following equation to the scribe.
Table 2. Audiometric responses at different frequencies of the three tested groups as mean and DS.

\begin{tabular}{cccc}
\hline $\begin{array}{c}\text { Frequencies } \\
(\mathrm{Hz})\end{array}$ & \multicolumn{3}{c}{ Groups } \\
\cline { 2 - 4 } & $\mathrm{A}-$ no smokers & $\mathrm{B}-$ smokers & $\mathrm{C}$ - heavy smokers \\
\hline 500 & $10,09 \pm 3,094$ & $11,06 \pm 2,05$ & $15,78 \pm 3,44$ \\
1000 & $11,84 \pm 4,015$ & $13,76 \pm 3,74$ & $18,58 \pm 5,05$ \\
2000 & $21,77 \pm 8,039$ & $26,91 \pm 5,31$ & $29,32 \pm 3,76$ \\
3000 & $33,3 \pm 10,11$ & $42,29 \pm 8,11$ & $46,32 \pm 9,26$ \\
4000 & $36,93 \pm 10,35$ & $53,76 \pm 8,22$ & $60,47 \pm 5,48$ \\
\hline
\end{tabular}

Table 3. Values of internal correlation coefficients for each group and five frequencies.

\begin{tabular}{cccc}
\hline Frequencies $(\mathrm{Hz})$ & Group A & Group B & Group C \\
\hline 500 & 5,1875 & 5,340277778 & 5,779861 \\
1000 & 5,93125 & 6,185416667 & 5,577778 \\
2000 & 6,185416667 & 5,691666667 & 5,857639 \\
3000 & 6,593055556 & 6,286805556 & 6,432639 \\
4000 & 6,670138889 & 6,479861111 & 5,8625 \\
\hline
\end{tabular}

The response trend for each audiometric frequency of the three tested groups were:

$$
\begin{aligned}
& 500 H z\left\{\begin{array}{lll}
G r(A) \Rightarrow R_{a}=3.0925 \times 1.2661^{x} & R^{2}=0.8089 \\
G r(B) \Rightarrow R_{a}=3.8716 \times 1.2382^{x} & R^{2}=0.8419 \\
G r(C) \Rightarrow R_{a}=8.5578 \times 1.1468^{x} & R^{2}=0.8277
\end{array}\right. \\
& 1000 H z\left\{\begin{array}{lll}
G r(A) & \Rightarrow R_{a}=3.0933 \times 1.3063^{x} & R^{2}=0.8033 \\
G r(B) \Rightarrow R_{a}=6.2183 \times 1.1884^{x} & R^{2}=0.8255 \\
\operatorname{Gr}(C) \Rightarrow R_{a}=7.7757 \times 1.2143^{x} & R^{2}=0.8354
\end{array}\right. \\
& 2000 H z\left\{\begin{array}{lll}
G r(A) & \Rightarrow R_{a}=4.8775 \times 1.3459^{x} & R^{2}=0.8572 \\
G r(B) \Rightarrow R_{a}=14.4558 \times 1.1464^{x} & R^{2}=0.8037 \\
G r(C) \Rightarrow R_{a}=20.1123 \times 1.0901^{x} & R^{2}=0.8614
\end{array}\right. \\
& 3000 \mathrm{~Hz}\left\{\begin{array}{lll}
\operatorname{Gr}(A) \Rightarrow R_{a}=11.0088 \times 1.2481^{x} & R^{2}=0.8146 \\
\operatorname{Gr}(B) \Rightarrow R_{a}=22.7629 \times 1.1465^{x} & R^{2}=0.9180 \\
\operatorname{Gr}(C) \Rightarrow R_{a}=24.2708 \times 1.1572^{x} & R^{2}=0.8840
\end{array}\right. \\
& 4000 H z\left\{\begin{array}{lll}
G r(A) & \Rightarrow R_{a}=13.3375 \times 1.2264^{x} & R^{2}=0.8091 \\
G r(B) \Rightarrow R_{a}=32.7116 \times 1.1167^{x} & R^{2}=0.9298 \\
G r(C) \Rightarrow R_{a}=46.2579 \times 1.0636^{x} & R^{2}=0.8496
\end{array}\right.
\end{aligned}
$$

The Figures 1-5 below show the theoretical trends determined by means of the aforementioned relation, by group, of the audiometric responses at the various frequencies, hypothesising a subject starting work at twenty years (average age of starting work found in each group):

The model proves that $I_{r}$ increase corresponds to increased hearing loss at each frequency in all tested groups.

The ANCOVA (Analysis of Covariance), applied to 


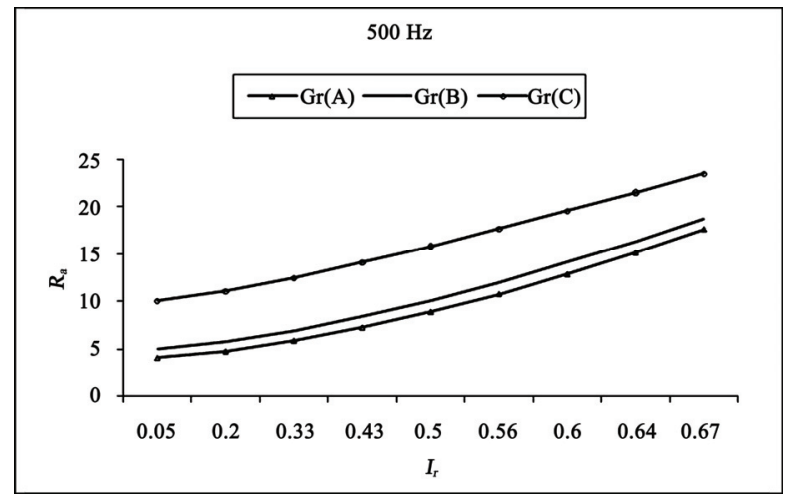

Figure 1. Theoretical trend of audiometric response at the frequency of $500 \mathrm{~Hz}$.

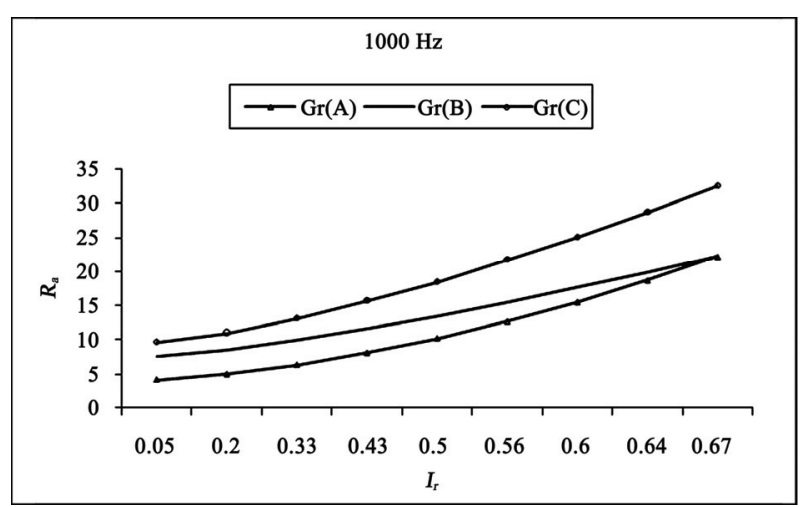

Figure 2. Theoretical trend of audiometric response at the frequency of $1000 \mathrm{~Hz}$.

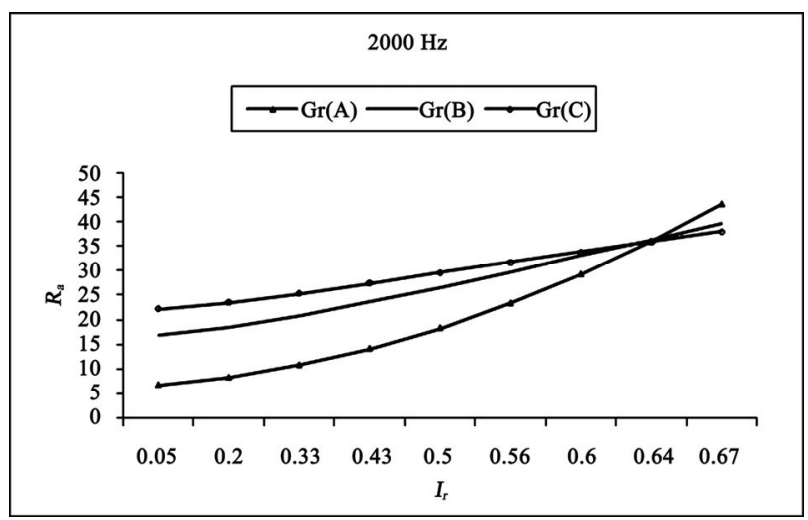

Figure 3. Theoretical trend of audiometric response at the frequency of $2000 \mathrm{~Hz}$.

the audiometric responses of the three groups, demonstrated that the trends of each group, for all the frequencies, may not be described by a single function, as can be seen from the results obtained by means of the parallelism test (Table 4).

The high significance of the p-values indicates that the relation between the variables studied is not sufficiently

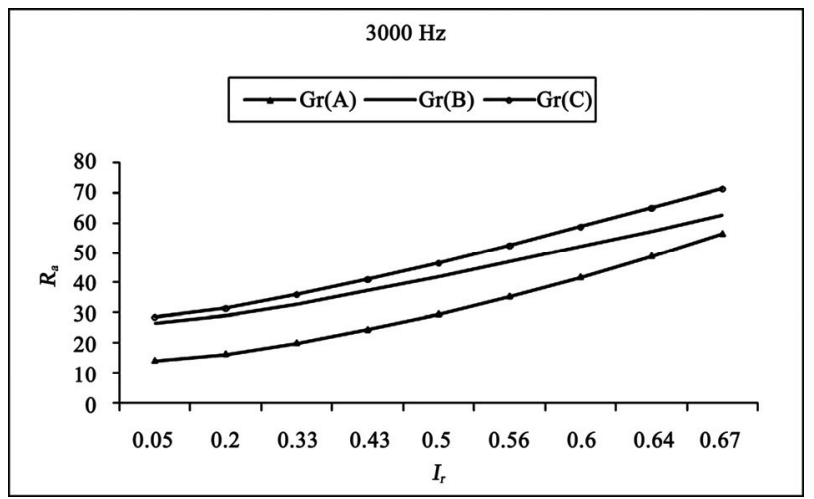

Figure 4. Theoretical trend of audiometric response at the frequency of $3000 \mathrm{~Hz}$.

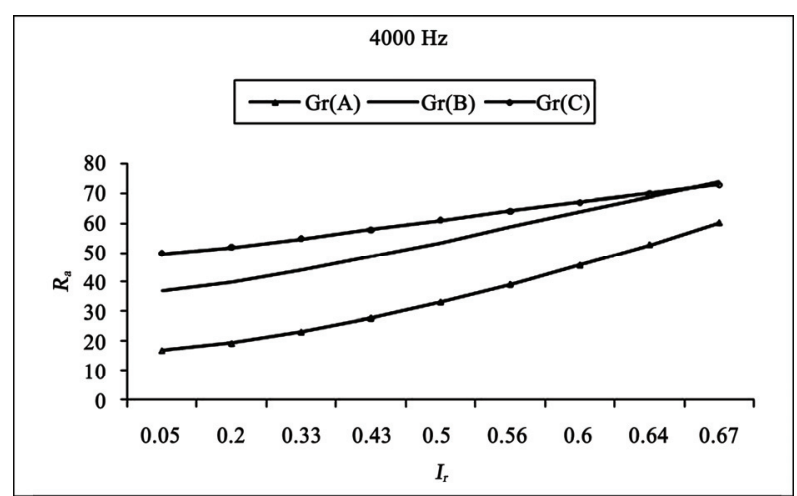

Figure 5. Theoretical trend of audiometric response at the frequency of $4000 \mathrm{~Hz}$.

Table 4. Results of the parallelism test.

\begin{tabular}{ccc}
\hline Groups & F & p-value \\
\hline ABC & 877.40 .00 & 0.000 \\
AB & 205.74 & 0.000 \\
AC & 12079.35 & 0.000 \\
BC & 16503.14 & 0.000 \\
\hline
\end{tabular}

represented by one single regression coefficient. Consequently, the three groups had to be considered separately.

The analysis thus far performed allowed us to demonstrate that the three groups, distinguished on the basis of smoking habits, underwent different variations in the temporal evolution of noise-induced hearing loss for all frequencies.

The results obtained led us to analyse the data in greater depth, in order to address a series of questions which naturally sprang to mind:

1) does smoking act generically on the audiometric responses, or only at specific frequencies?

2) are the audiometric responses equally influenced in the two groups of smokers?

3) do age and occupational exposure influence the two 
groups compared differently?

For this reason, the $I_{r}$ for the whole sample was stratified in 10 layers using $I_{r}$ distribution percentiles.

We stratified $I_{r}$ relative to all subjects, in 10 layers using $I_{r}$ distribution percentiles. Table 5 shows $I_{r}$ values.

We conducted a comparative analysis for layers and each frequency of $R_{a}$, between the three groups. The method used was the Nonparametric test Combination (NPC test), that provides effective solutions for problems of multidimensional hypothesis testing within nonparametric permutations, and is used to test multidimensional hypotheses which are too complex to manage parametrically.

Consequently, we conducted a comparative nonparametric analysis of audiometric responses among the three groups for each layer and all frequencies, using the NPC test. The results obtained, showing that the null hypothesis is not verified for both partials and combined test, prove that relating to cigarettes smoking the three groups are significantly different.

The test results highlighted that the three groups are different for all the frequencies and all the layers $(\mathrm{p}=$ $0.000)$.

But are the results attributable to the $R_{a}$ values for all the frequencies of the three groups, or are just some of these, and only for some frequencies, the cause of the significant differences between non-smokers and more or less heavy smokers?

We thus performed comparisons in pairs of the $R_{a}$ values between the groups of subjects, by layer and for all the frequencies.
The hypothesis for the comparison between one group $(X)$ and another $(Y)$, homogeneous in terms of age and length of exposure, is as follows:

$$
\begin{aligned}
& H_{0 i\left(F r_{j}\right)}:\left\{\left(R_{a} G r(X) \stackrel{d}{=} R_{a} G r(Y)\right\} \text { with } j\right. \\
& =500 H z, \ldots \ldots . . ., 4000 \mathrm{~Hz}
\end{aligned}
$$

As far as regards the comparison between non-smokers and smokers, the test showed that the $\mathrm{R}_{\mathrm{a}}$ values in the two groups were not differentiated for the frequency of $500 \mathrm{~Hz}$ from the fourth layer of the risk index upwards (average length of exposure of 17 years), while for the frequencies 1000 and $2000 \mathrm{~Hz}$ from the eighth layer upwards (average length of exposure of 27 years) (Table 6).

Table 5. $I_{r}$ distribution percentiles.

\begin{tabular}{ccc}
\hline \multirow{2}{*}{ Percentiles } & \multicolumn{2}{c}{$I_{r}$ Values } \\
\cline { 2 - 3 } & Minimum & Maximum \\
\hline $10^{\circ}$ & 1,519444444 & 2,231944444 \\
$20^{\circ}$ & 2,240277778 & 2,634027778 \\
$30^{\circ}$ & 2,670833333 & 3,002777778 \\
$40^{\circ}$ & 3,009027778 & 3,279166667 \\
$50^{\circ}$ & 3,289583333 & 3,472222222 \\
$60^{\circ}$ & 3,545833333 & 3,684722222 \\
$70^{\circ}$ & 3,69375 & 3,941666667 \\
$80^{\circ}$ & 3,945833333 & 4,119444444 \\
$90^{\circ}$ & 4,133333333 & 4,323611111 \\
$100^{\circ}$ & 4,340277778 & 4,728472222 \\
\hline
\end{tabular}

\begin{tabular}{|c|c|c|c|c|c|c|c|}
\hline \multirow{2}{*}{ Layers } & \multicolumn{5}{|c|}{ Audiometric Responses } & & \multirow[b]{2}{*}{ T.C. } \\
\hline & $500 \mathrm{~Hz}$ & $1000 \mathrm{~Hz}$ & $2000 \mathrm{~Hz}$ & $3000 \mathrm{~Hz}$ & $4000 \mathrm{~Hz}$ & & \\
\hline$I_{r 1}$ & 0.000 & 0.000 & 0.000 & 0.000 & 0.000 & $\rightarrow$ & 0.000 \\
\hline$I_{r 2}$ & 0.000 & 0.004 & 0.000 & 0.000 & 0.000 & $\rightarrow$ & 0.000 \\
\hline$I_{r 4}$ & 0,343055556 & 0.000 & 0.000 & 0.000 & 0.000 & $\rightarrow$ & 0.000 \\
\hline$I_{r 5}$ & 0,3125 & 0.000 & 0.000 & 0.000 & 0.000 & $\rightarrow$ & 0.000 \\
\hline$I_{r 6}$ & 0,421527778 & 0.003 & 0.000 & 0.000 & 0.000 & $\rightarrow$ & 0.000 \\
\hline$I_{r 7}$ & 0.069 & 0.000 & 0.005 & 0.000 & 0.000 & $\rightarrow$ & 0.000 \\
\hline$I_{r 9}$ & 0,229861111 & 0.067 & 0,072917 & 0.000 & 0.000 & $\rightarrow$ & 0.000 \\
\hline \multirow[t]{3}{*}{$I_{r 10}$} & 0,141666667 & 0,122916667 & 0,266667 & 0.020 & 0.000 & $\rightarrow$ & 0.000 \\
\hline & $\downarrow$ & $\downarrow$ & $\downarrow$ & $\downarrow$ & $\downarrow$ & & $\downarrow$ \\
\hline & 0.000 & 0.000 & 0.000 & 0.000 & 0.000 & & 0.000 \\
\hline
\end{tabular}

Table 6. Results of the comparison between non-smokers and smokers. 


\section{DISCUSSION}

The data obtained from the sample under study demonstrated that cigarette smoking interferes with the onset and development of hearing loss caused by industrial noise.

The results of the statistical study applied to the audiometric responses displayed statistically significant differences between non-smokers, smokers and heavy smokers.

This demonstrates that smoking has an influence on noise-induced hearing loss and that this effect is correlated to the quantity of cigarettes smoked daily.

The theoretical model applied for each frequency on the distribution trend of the values of $R_{a}$ highlighted that, with increased age and length of service, the audiometric responses for all frequencies increase in all three groups, albeit in a differentiated manner.

Furthermore, the ANCOVA(Analysis of Covariance) results show that the trends of the $R_{a}$ values for the three groups may not be described using a model with the same parameters.

The results of the non-parametric analysis demonstrated that the three groups examined are differentiated, in relation to the audiometric responses for all the frequencies and for each layer.

In particular it was found that the risk factor influences all frequencies, and especially those at the higher end of the spectrum.

These observations are in accordance with the findings of Mizoue et al. [8] who, in a study on 4624 steel workers exposed to noise, highlighted an increase in noiseinduced damage mainly at high frequencies, in relation to the quantity of cigarettes smoked.

Our result agree with Wild's ones [15] that showed noise-inducted hypoacusis increase at $3000-4000 \mathrm{~Hz}$, in a group of long time smokers compared with no smokers with similar occupational history.

Our data show effects at low frequencies, not found by Mizoue et al. [8], and this observation is due to the different sensitivity of the statistical techniques used, and in particular in the use of the NPC test.

Regarding the comparison between smokers and heavy smokers, the statistical study, which highlighted significantly different behaviour for all the frequencies, confirmed, as reported by the studies of Nakanishi et al. [16] and Mizoue et al. [8], that the effects of cigarette smoking have an effect on noise-induced hearing loss which depends on the quantity of cigarettes smoked per day.

The pathogenetic mechanisms involved may be identified as:

1) the ototoxic effect performed by nicotine, which stimulates the nicotine receptors of the acoustic cells as described by Evans [17] and by Blachet et al. [18].
2) the mechanisms correlated to ischaemic damage caused by decreased blood flow. In fact, the physiopathology of hearing loss involves a reduction in cochlear blood flow and gaseous exchange, and contemporaneous increased levels of circulating $\mathrm{CO}$ caused by chronic exposure to cigarette smoking further reduces the quantity of oxygen to the cochlear cells, thus contributing to sensory neural damage. This hypothesis, suggested by Matschke [19] in acute noise-induced damage, was subsequently confirmed by the studies of Fechter et al. [20-22], Rao et al. [23] and Shahbaz Hassan et al. [24] for chronic noise-induced damage. Rao et al. [23], in an experimental study, highlighted that the simultaneous exposure to noise and $\mathrm{CO}$ increases noise-induced hearing loss. Fechter et al. [22], in an experimental study on laboratory animals, demonstrates that $\mathrm{CO}$ increases noise-induced hearing loss. Shahbaz Hassan et al. [24] reaches the same conclusions, claiming that $\mathrm{CO}$ increases noiseinduced hearing loss.

\section{CONCLUSIONS}

Our data confirm the aforementioned hypotheses also in conditions of chronic exposure.

In conclusion, the observations deriving from the study show that cigarette smoking acts in synergy with exposure to industrial noise on hearing loss, and that this effect is correlated to the amount of cigarettes smoked. Thus, we feel it is necessary to encourage workers occupationally exposed to high levels of noise to limit cigarette smoking.

\section{REFERENCES}

[1] Cruickshanks, K.J., Klein, R., Klein, B.E., Wiley, T.L., Nondahl, D.M. and Tweed, T.S. (1998) Cigarette smoking and hearing loss: The epidemiology of hearing loss study. Journal of the American Medical Association, 279(21), 1715-1719.

[2] Starck, J., Toppila, E. and Pyykko, I. (1999) Smoking as a risk factor in sensory neural hearing loss among workers exposed to occupational noise. Acta Otolaryngology, 119(3), 302-305.

[3] Itoh, A., Nakashima, T., Arao, H., Wakai, K., Tamokoshi, A., Kawamura, T. and Ohno, Y. (2001) Smoking and drinking habits as risk factors for hearing loss in the elderly: Epidemiological study of subjects undergoing routine health checks. Aichi Japan Public Health, 115(3), 192-196.

[4] Linke, R. and Matschke, R.G. (1998) Is there a correlation between sudden deafness and smoking? Laryngorhinootologie, 77(1), 48-51.

[5] Sharabi, Y., Reshef-Haran, I., Burstein, M. and Eldav, A. (2002) Cigarette smoking and hearing loss: Lessons from the young adult periodic examinations in Israel (YAPEIS) database. Israel Medical Association Journal, 4(12), 
$1118-1120$.

[6] Nomura, K., Nakao, M. and Morimoto, T. (2005) Effect of smoking on hearing loss: Quality assessment and meta-analysis. Preventive Medicine, 40(2), 138-144.

[7] Palmer, K.T., Griffin, M.J., Syddall, H.E. and Coggon, D. (2004) Cigarette smoking, occupational exposure to noise, and self reported hearing difficulties. Occupational and Environmental Medicine, 61(3), 340-344.

[8] Mizoue, T., Miyamoto, T. and Shimizu, T. (2003) Combined effect of smoking and occupational exposure to noise on hearing loss in steel factory workers. Occupational and Environmental Medicine, 60(1), 56-59.

[9] Dengerink, H.A., Lindgren, F.L. and Axelsson, A. (1992) The interaction of smoking and noise on temporary threshold shifts. Acta Otolaryngology, 112(6), 932-938.

[10] Nomura, K., Nakao, M. and Yano, E. (2005) Hearing loss associated with smoking and occupational noise exposure in a Japanese metal working company. International Archives of Occupational and Environmental Health, 78(3), 178-184.

[11] Jaruchinda, P., Thongdeetae, T., Panichkul, S. and Hanchumpol, P. (2005) Prevalence and analysis of noise induced hearing loss in army helicopter pilots and aircraft mechanisms. Journal of the Medical Association of Thailand, 88(Suppl. 3), 232-239.

[12] Pouryaghoub, G., Mehrdad, R. and Mohammadi, S. (2007) Interaction of smoking and occupational noise exposure on hearing loss: A cross-sectional study. $B M C$ Public Health, 7, 137-143.

[13] Pyykko, I., Koskimies, K., Starck, J., Pekkarinen, J., Farkkila, M. and Inaba, R. (1989) Risk factors in the genesis of sensory neural hearing loss in Finnish forestry workers. British Journal of Industrial Medicine, 46(7), 439-446.

[14] Nakashima, T., Tanabe, T., Yanagiha, N., Wakai, K. and Ohno, Y. (1997) Risk factors for sudden deafness: A case control study. The International Journal Auris Nasus
Larynx, 24(4), 265-270.

[15] Wild, D.C., Brewster, M.J. and Banerjee, A.R. (2005) Noise induced hearing loss in exacerbated by long-term smoking. Clinical Otolaryngology, 30(6), 517-520.

[16] Nakanishi, N., Okamoto, M., Nakamura, K., et al. (2000) Cigarette smoking and risk for hearing impairment: A longitudinal study in Japanese male office workers. Occupational and Environmental Medicine, 42(11), 1045 1049.

[17] Evans MG (1996) Acetylcholine activates two currents in guinea-pig outer hair cells. Journal of Physiology, 1(491), 563-578

[18] Blachet, C., Erostegui, C., Sugasawa, M. and Dulon, D. (1996) Acetylcholine-induced potassium current of guinea pig outer hair cells: Its dependence on a calcium influx through nicotinic-like rereptors. Neurosciences, 16(8), 2574-2584.

[19] Matschke, R.G. (1990) Smoking habits in patients with sudden hearing loss. Preliminary results. Acta Otolaryngology, 476(Suppl.), 69-73.

[20] Fechter, L.D., Chen, G.D., Rao, D., et al. (2000) Predicting exposure conditions that facilitate the potentietion of noise - induced hearing loss by carbon monoxide. Toxicology Sciences, 58(2), 315-323.

[21] Fechter, L.D., Chen, G.D. and Rao, D. (2002) Chemical asphyxiants and noise. Noise Health, 4(14), 49-61.

[22] Fechter, L.D., Cheng, G.D. and Rao, D. (2003) Characterising conditions that favour potentiation of noise induced hearing loss by chemical asphyxiants. Noise Health, 3(9), 11-21.

[23] Rao, D.B. and Fechter, L.D. (2000) Increased noise severity limits potentiation of noise induced hearing loss by carbon monoxide. Hearing Research, 150(1-2), 206-214.

[24] Shahbaz Hassan, M., Ray, J. and Wilson, F. (2003) Carbon monoxide poisoning and sensorineural hearing loss. Journal of Laryngology \& Otology, 117(2), 134-137. 